\title{
Juventudes y TIC: Estados locales frente al abordaje de la promoción de la lectura
}

\section{Pereyra, Eduardo}

Resumen:

El presente artículo pone en discusión una serie de afirmaciones en torno a la supuesta "crisis de lectura" en las juventudes; las miradas clásicas respecto a qué, cómo y cuándo los jóvenes deben leer y respecto a los paradigmas más clásicos y conservadores sobre cómo se define y aborda la cuestión juvenil desde el Estado. A partir de ello, el objetivo central es dar relevancia a la capacidad de los Estados locales en el abordaje las tecnologías de la información y la comunicación (TIC) para intervenir en la promoción de la lectura implementando políticas públicas juveniles que se orienten a la generación de condiciones para que los jóvenes puedan autorealizarse y a su vez puedan participar en la construcción de la realidad social en la que se insertan.

Palabras clave: Juventudes - TIC - Promoción de la Lectura - Estado Local - Políticas Públicas.

Magister en Diseño y Gestión de Programas Sociales por la Facultad Latinoamericana de Ciencias Sociales (FLACSO - Argentina) y Licenciado en Ciencia Política por la Universidad Católica de Córdoba. Becario CONICET y Doctorando en Política y Gobierno (UCC). Investigador y consultor en políticas públicas en materia de cuestión social y juvenil. Trayectoria profesional en gestión pública y docencia de nivel medio.

Introducción

Desde ámbitos académicos e institucionales se viene sosteniendo que se atraviesa una fuerte "crisis de lectura", particularmente en la población juvenil. Desde estos mismos ámbitos se pone en debate el rol de la escuela y el incentivo a la lectura, aunque desde miradas mayormente tradicionales. Sin embargo existe en los últimos años profusas investigaciones que ponen en discusión estas miradas y la noción de crisis y asumen el desafío de 
incorporar, con todas sus complejidades, el mundo digital-audiovisual al diseño de políticas de lectura. A su vez, este artículo1 propone atravesar esta discusión con el rol del Estado local en la temática y los paradigmas que se asumen sobre la cuestión juvenil para avanzar en la implementación de políticas municipales integrales y acordes a los desafíos actuales de la problemática. Para ello se vuelve fundamental atender los nuevos roles de los gobiernos locales, el abandono de paradigmas residuales sobre la juventud con la consecuente incorporación transversal del enfoque de derechos y finalmente aplicar las nociones de Descentramiento y Destemporalización (Barbero, 2003) adoptadas por la “Educación en medios" (Morduchowicz, 2009) a las políticas de promoción de la lectura.

A tal fin, en primer lugar, se presentarán algunos aspectos ligados a la consideración de la reconfiguración de los problemas públicos en agenda de los Estados Locales. En segundo lugar, se hará referencia a las definiciones en torno a las juventudes y la cuestión juvenil, su ingreso en la agenda estatal y los paradigmas preponderantes en las políticas gubernamentales para el sector. En tercer lugar, se analizará la compleja relación entre juventud, las tecnologías de la información y de la comunicación (TIC) y promoción de la lectura para finalizar con algunas reflexiones en torno a las consideraciones de atender por parte del Estado local el abordaje de tal compleja ecuación.

1. Estado local y la nueva agenda "recargada" de problemas públicos

En los debates contemporáneos acerca de las políticas de lectura (Bombini, 2008; Mihal, 2009; 2012, entre otros), la mirada ha estado centrada en el Estado desde el nivel nacional o en instituciones particulares tales como bibliotecas, escuelas, cárceles, entre otros espacios. Sin embargo es mucho menor la atención recibida en los estudios en los propios municipios, o cuando tales investigaciones se han centrado en las políticas culturales (Calabre, 2009; Calado, 2012; Rebón, 2011), por ejemplo, salvo algunas referencias acerca de las bibliotecas, la lectura no ocupado un lugar predominante en los mismos.

No obstante ello, el Estado local se ha configurado en un actor clave para el desarrollo de políticas públicas. En este sentido, a partir de las Reformas del Estado ejecutadas en Argentina, durante la década del '90, se determinaron nuevas funciones y alcances de los municipios (Arroyo, 1997; Cippec, 2005; García Delgado, 1997; Maceira, et.al, 2010), cambiando las relaciones de los niveles nacionales, provinciales y municipales.

A las funciones tradicionales de gestión administrativa, prestación de servicios públicos, desarrollo urbano y regulación de la vida comunitaria, los municipios debieron asumir un rol protagónico en la provisión de bienes y servicios públicos que ubican al Estado local como un actor estratégico en la planificación del desarrollo económico, social y cultural de la ciudad. En función de esto, los municipios se vieron obligados a transformar cuestiones claves respecto a su autonomía, organización e implementación de políticas públicas. Debieron incorporar entonces, acciones referidas a: promoción económica y desarrollo productivo, empleo, gestión cultural, educación, cuidado del medio ambiente, desarrollo social, defensa de derechos ciudadanos y consumidores, seguridad, la cuestión juvenil y salud. Ahora bien, dichas incorporaciones según García Delgado (1997) se vinculan con tres cambios producidos en: 1) lo organizativo y político-institucional -con la introducción del modelo gerencial de la Nueva Gestión Pública (NGP) con los criterios de eficiencia, eficacia y gestión por resultados, desplazando el modelo burocrático-; 2) lo económico -debido al ajuste y dificultades financieras, los municipios se vieron obligados a un mayor activismo económico. Se incorporó a la gestión local las nociones de capacidad y planificación estratégica, avanzando en el diseño e implementación de políticas de empleo, 
promoción de la inversión y generación de infraestructura, y lo social-; y 3) la cuestión social -también bajo el paradigma dominante de la NGP-, comenzó a ser un tema instalado en la agenda pública local.

El supuesto central, sobre el que se sustentaron estos cambios, es que la instancia gubernamental local es un espacio privilegiado de cercanía y contacto con la población para poder abordar los problemas con mayor eficacia y eficiencia. Con lógicas de articulación y fortalecimiento de la sociedad civil, la gestión ejecuta políticas focalizadas, de financiamiento a la demanda, con creación de consejos y talleres, innovando en la introducción de sistemas de monitoreos, control y evaluación de los nuevos roles y acciones asumidos.

Sin embargo, es posible advertir que hasta el presente, existen una serie de dificultades estructurales que atraviesan los municipios en la Argentina en cuanto a sus habilidades y capacidades de gestión2 (Cravacuore, 2007). Para el caso puntual de este trabajo, las principales dificultades que atraviesan los municipios en materia de políticas de lectura se vinculan con las bajas o nulas capacidades estatales para el abordaje de la promoción de la lectura, lo cual se puede visualizar en una marcada ausencia de presupuestos destinados a la promoción de la lectura; la poca o baja presencia de personal municipal especializado en la materia y; la carencia de información sistemática respecto a los consumos culturales juveniles locales y su relación con las TIC, es decir, con los entrecruzamientos de la lectura en otros soportes además de libros, en la órbita de los municipios. Asimismo, generalmente las principales acciones en promoción de lectura se concentran en bibliotecas públicas, que no necesariamente son municipales pues pueden formar parte de instituciones del Estado provincial, no del local; o ser bibliotecas populares (adscriptas a la Comisión Nacional de Protección de Bibliotecas Populares- CONABIP, organismo dependiente del Estado nacional), o, estar en la órbita de las escuelas, lo que implica que quedan excluidos de ellas los sectores fuera del sistema educativo formal, -dado que la mayoría de los proyectos tienen como principal población objetivo a los que se encuentran realizando una trayectoria institucional-.

En suma, a partir de estas dificultades identificadas se puede observar que los gobiernos locales tienen una muy baja incidencia en la problemática. Asimismo, en los municipios conviven miradas difusas y contradictorias respecto a cómo definir a la población joven y el abordaje de la cuestión juvenil. Esto atenta fuertemente contra las posibilidades de elaborar una política integral de lectura que tenga en cuenta los consumos culturales de los jóvenes, inclusive aquellos consumos vinculados con las TIC, pero sobre todo los derechos de las juventudes en esta materia.

Cabe destacar, que dichas miradas también dan cuenta de las dificultades de definir en la actualidad qué se entiende por juventudes, como se verá a continuación.

2. Juventudes, cuestión juvenil y su abordaje estatal

2.1 Interprentando lo juvenil

Numerosos autores han contribuido al debate y análisis de la categoría juventud desde diferentes perspectivas. Al respecto, Quapper (2001) parte de la clasificación de cuatro versiones respecto a lo juvenil. En primer lugar, la definición más clásica que determina a la juventud como una etapa de la vida: por un lado, momento distinguible dentro del ciclo vital (niñez, adultez y vejez); por otro lado, como una etapa de preparación para ingresar al 
mundo adulto. Esta visión de la juventud como "tránsito hacia" se relaciona con el concepto de moratoria social, Braslavsky sostiene que se trata:

...de una serie de transiciones paralelas o consecutivas que varían histórica y culturalmente (...) algunos autores consideran cinco transiciones: 1) dejar la escuela; 2) comenzar a trabajar; 3) abandonar el hogar de la familia de origen; 4) casarse y 5) establecer un nuevo hogar. (Braslavsky, 1986, p. 12)

En segundo lugar, la juventud como referencia a un grupo social distinguible por determinadas variables, centralmente lo etario. La tercera versión refiere a la juventud en relación con una postura, actitud ante la vida. Lo juvenil es vitalidad, alegría, capacidad de cambio, de (re)aprender el mundo, de tener todo un futuro por delante. Y, por último, los jóvenes como generación futura, que al convertirse en adultos llevarán las riendas de la sociedad para garantizar su reproducción.

Ahora bien, el autor visualiza estas definiciones como conservadoras y fundamentalmente adultocéntricas, es decir que todas parten de la definición que el mundo adulto hace de lo juvenil y por ende determina qué es y qué se espera de esta generación. Así entendido, afirma que estas versiones generan trampas para poder comprender de manera compleja e integral el mundo de las y los jóvenes.

La primera trampa tiende a definir la juventud de manera universalizadora y homogeneizadora. La juventud es una o todos los jóvenes son iguales sin distinción alguna en relación a géneros, clase sociales, estilos culturales, etc. La segunda trampa parte de generar una estigmatización del grupo social juventud, donde es visto como un problema o bien sujeto con problemas, que obstaculiza el desarrollo "normal" de la vida en sociedad: orden, paz y progreso (Quapper, 2001). En definitiva, la juventud se asocia con lo patológico. La tercera trampa es la invisibilización de la complejidad de lo juvenil. Si la juventud es vista simplemente como una etapa del ciclo vital, se produce un encasillamiento determinista donde las personas son una cosa y luego otra: por ejemplo, se es joven o adulto, sin posibilidad de que exista un complejo entramado de convivencias dentro del mismo ciclo vital. Por último, la cuarta trampa genera una idealización normativista del (deber ser) joven. Los aspectos positivos de lo juvenil es que ellos son el futuro, los que deben transformar la realidad. Son la esperanza y tienen la responsabilidad, por el hecho de ser jóvenes, de formarse para "salvar el mundo" (Quapper, 2001).

Un aporte fundamental sobre las representaciones y visiones predominantes y vigentes sobre las y los jóvenes urbanos de la Argentina actual, es el trabajo de Chaves (2005), en el cual sostiene que:

... las miradas hegemónicas sobre la juventud latinoamericana responden a los modelos jurídico y represivo del poder (...) la juventud está signada por <>, es negada (modelo jurídico) o negativizada (modelo represivo), se le niega existencia como sujeto total (en transición, incompleto, ni niño ni adulto) o se negativizan sus prácticas (juventud problema, juventud gris, joven desviado, etc.). (Chaves, 2005, p. 26)

Además, la autora identifica seis discursos que entienden de distinto modo a la juventud y los jóvenes, el naturalista (como etapa natural, biológica y universal); el psicologicista (como una etapa de confusión que debe transitar y que sí o sí pasará); el de la patología social (como parte del cuerpo social, se encuentra enfermo y representa un problema a la sociedad; el del pánico moral (como desviados y peligrosos generando miedo a la comunidad); el culturalista: (como grupo etario que puede construir cultura por sí misma, transmitiendo y reproduciéndola a otras generaciones; y el sociologista (como producto determinado por los parámetros 
establecidos en la sociedad en la cual vive). En este sentido, Chaves (2005) sostiene que estos discursas le dan fundamento a las representaciones antes citadas, invisibilizando a los jóvenes, anulando sus visiones, pensamientos y sentimientos y, fundamentalmente, negando su capacidad de acción y participación en la dinámica de la realidad social.

Margulis (2001), por otra parte, remite a dos tipos de definición de lo juvenil. Uno bioló- gico-etario y otro de carácter histórico. El primero es "tradicional" y conservador, estático y positivista, define a las y los jóvenes como un momento en el desarrollo biológico-humano; se identifica con lo etario, una etapa en la vida corporal que "debe" transcurrir para llegar a "ser" adulto. El segundo, es un concepto histórico que adquiere su especificidad según la construcción social que se da: asocia lo juvenil con un sector social que construye su identidad en el marco de las relaciones sociales e institucionales, que son históricamente construidas y determinadas. En esta segunda tipología de lo juvenil encuentra tres variables: la diferenciación social, el género y la generación.

En definitiva, para Margulis las variables género, generación y clase permiten ya no hablar a través del concepto monolítico y uniforme de juventud, sino que se hace necesario entender e interpretar las diversidades existentes en función del plano histórico, social, económico y cultural, las diferentes juventudes que conviven aún en la misma realidad social. Quapper comparte y completa este cambio de concepción de juventud a juventudes siendo necesario: mirar y comprender las singularidades, pluralidad y diversidad de juventudes en cada espacio social; advertir la existencia de subgrupos etarios que son de referencia para la categorización de las juventudes; vislumbrar la riqueza y complejidad de lo juvenil a través de una mirada compleja ("caleidoscópica", en términos del autor); interpretar al mundo juvenil como múltiple y diverso y a partir de ello generar conocimiento y conceptos dinámicos, flexibles que permitan acercarnos, abordar y comprender a las y los jóvenes, las juventudes, las expresiones juveniles y los procesos de juvenilización3 (Quapper, 2001). En suma, estos diferentes autores cuestionan una definición unívoca y estática de la juventud, poniendo en debate los principales paradigmas adoptados durante décadas por el Estado en sus distintos niveles, y que aún perdura en diversas dependencias gubernamentales, para el diseño e implementación de políticas. Estas nuevas categorías teóricoconceptuales permitieron a su vez, el surgimiento de renovados paradigmas y que a continuación analizaremos en su conjunto.

\subsection{Cuestión juvenil en la agenda estatal y paradigmas de abordaje}

El concepto de juventud es producto de las revoluciones burguesas y la conformación de las sociedades capitalistas. Son los conflictos surgidos de las relaciones sociales, de poder y de producción los que dan nacimiento a este nuevo actor social (Balardini, 2000). Puntualmente, la categoría de "lo juvenil" surge en las modernas sociedades industriales a partir de la expansión del trabajo intelectual asalariado y para ello es necesario la formación de este tipo de asalariados, viendo en los jóvenes de clase media y alta el futuro asalariado y por ende la necesidad de su capacitación acorde dentro del sistema educativo. (Saltamacchia, 1990)

Generalmente la noción de juventud, como se ha visto en el apartado anterior, al igual que las nociones de niñez o vejez, está fuertemente asociada a una determinada edad de las personas. Sin embargo, las clasificaciones etarias, como las de sexo o clase, son formas de imponer límites en pos de producir y sostener un orden social determinado. En ese sentido cada campo tiene sus leyes específicas de envejecimiento, y la edad constituye un dato biológico socialmente manipulado y manipulable (Bourdieu, 2002, p. 164). 
Los ámbitos de socialización por excelencia durante las sociedades burguesas estuvieron determinados por tres instituciones que surgieron en la época para tales fines: la escuela, la familia y la niñez. Sin éstas, la juventud como tal no habría surgido:

Si lo dijéramos en términos fabriles (y la escuela sería aquí la fábrica), cada vez se va a producir más ; la escuela es el espacio privilegiado de producción de juventud (...) poco a poco y con el tiempo, se van a ir integrando nuevos contingentes de individuos jóvenes al sistema escolar productor de juventud. (Balardini, 2000, p. 14)

La escuela es el campo central de producción y formación de la juventud. Por ello, durante todo el siglo XIX la asociación de jóvenes (de las clases altas y medias) con estudiantes es la principal definición de sujeto de políticas públicas por parte del Estado moderno hacia el sector. Luego a principios del siglo XX aparecerá la "juventud trabajadora" y más adelante aparecerán otras categorizaciones como la "juventud rural” (Balardini, 2000).

Las crisis sociales, políticas y económicas vividas en los países capitalistas en la primera mitad del siglo XX, con las profundas consecuencias de la primera y segunda guerra mundial, junto a los desplazamientos y migraciones poblacionales y las revoluciones tecnológicas, amplían y diversifican las nociones de juventud, no sólo en asociación con un sector determinado (jóvenes estudiantes, jóvenes trabajadores, jóvenes rurales, jóvenes urbanos, etc.) sino más bien a un sujeto con determinada edad que es poseedor de derechos y deberes. En este sentido es con el Estado de Bienestar que comienzan a diseñarse e implementarse políticas públicas estatales que buscan responder ante las diversas problemáticas que atañen exclusivamente a este sector poblacional de nuestras sociedades.

Desde la década de 1980 se asiste a lo que se ha denominado una "nueva cuestión social" (Golbert, 2004; Rosanvallon, 1995), surgida tras los quiebres ocurridos a partir de 1970 en el orden financiero, ideológico y filosófico que han puesto en crisis al Estado de Providencia. Esto supone un cambio en las concepciones mediante las cuales el Estado, las diversas instituciones y la sociedad entera comprendían las problemáticas sociales de la época y definían cómo y quiénes debían hacerse responsables de su resolución.

Por lo tanto, desde 1980, al instalarse la nueva cuestión social, el que ha entrado en crisis ha sido el Estado de Providencia con su sistema de solidaridad y derechos sociales. Es en este contexto que la temática de la juventud cobra relevancia en la agenda de los Estados nacionales, como el caso argentino.

Asimismo Ernesto Rodriguez indica que el contexto de exclusión social estructural, pos crisis de los '70, ha sido el principal problema a abordar por las políticas estatales, inclusive las respuestas de las instituciones responsables del diseño e implementación de políticas juveniles pecan de la ineficiencia e ineficacia. Los errores provienen -según el mismo autor- de la configuración de determinados "modelos" de políticas de juventud, a saber: el más antiguo, que se centra en la educación y tiempo libre de los jóvenes; los centrados en el control social de jóvenes movilizados y de la pobreza como método de combate al delito; y desde mediados de los '90, el modelo de inserción laboral juvenil basado en la "inversión del capital humano". (Rodriguez, 2000, p. 29)

Entre las características comunes de estos modelos, el autor indica la pretensión de universalidad de las políticas, la excesiva burocratización del Estado para su desarrollo y que, al contrario de las urgencias sociales 
juveniles, las políticas eran destinadas mayoritariamente a los "jóvenes integrados". Los modelos, siguiendo al autor, apuntaban a la preparación de los jóvenes para su ingreso al mundo adulto a través de la educación, funcionalizando "adecuadamente" la utilización del tiempo libre, y definiendo la juventud como "la esperanza del futuro".

En relación a la identificación de modelos preponderantes en la concepción del Estado respecto a los jóvenes y en función de esos paradigmas, las políticas y programas que el mismo Estado diseña e implementa, Dina Krauskopf (2000) distingue los siguientes:

El paradigma "Periodo preparativo - transición a la adultez" entiende a los jóvenes como inmaduros e inexpertos, se encuentran "incompletos". Por ende no se los considera como un actor social, avalando el deber de la intervención adulta en sus vidas. La juventud es un periodo de ensayo y transición para "aprender" a ser adultos “... es la etapa de la vida dedicada esencialmente a la adquisición de conocimientos. Para ello, la sociedad otorga una moratoria de roles, esto es, una suspensión temporal de obligaciones..." (Krauskopf, 2000, p. 10). En términos de políticas públicas, este paradigma se tradujo en la universalización del sistema educativo. El segundo paradigma, la juventud como "etapa problema", da importancia a la temática juvenil a partir de las perturbaciones que generan los "problemas" que poseen los jóvenes y que alteran el orden social. Entran en la agenda estatal las cuestiones de delincuencia juvenil, deserción escolar o de cuestiones de salud como embarazos adolescentes, enfermedades de transmisión sexual y adicciones de sustancias legales e ilegales. Esto implica que las políticas adoptadas deben generar un control socialadulto sobre los jóvenes, de represión o rehabilitación de los mismos, generando una estigmatización negativa y muchas veces criminalizante de la juventud. El tercer paradigma, la "juventud ciudadana", se encuentra en contraposición de la visión estigmatizante sobre los jóvenes, considerando a los mismos como un actor social que debe participar en la construcción de la calidad de vida en democracia y en el desarrollo de su sociedad. Sin embargo este enfoque, que intenta abandonar el predominio del discurso adultocéntrico tradicional a la hora del diseño e implementación de políticas públicas, ha generado ciertas dudas respecto a la definición de participación, provocando un ejercicio restringido de la ciudadanía por parte de los jóvenes, ya que los mismos se ven imposibilitados de intervenir de manera activa, como sujeto de derechos, en la planificación y toma de decisión de las políticas destinadas a su sector.

Considerando que los dos primeros modelos han fracasado en sus objetivos y no han podido aportar eficaz y eficientemente al abordaje de la problemática juvenil, más las contradicciones generadas del tercer modelo, los autores mencionados proponen respuestas focalizadas basadas en los jóvenes como "actores estratégicos del desarrollo" (Rodríguez, 2000, 2003; Krauskopf, 2000). En este sentido, Krauskopf (2000) señala que es durante la década de los 90 cuando se marca un hito en la región, tras la incorporación del enfoque de derechos en el diseño de políticas públicas, fundamentalmente en el campo de la niñez y adolescencia, trasladándose poco a poco a otros ámbitos como el juvenil.

El enfoque de derechos considera al derecho internacional sobre los derechos humanos como un marco conceptual que permite definir que el punto de partida para las políticas públicas no sea la existencia de personas con necesidades insatisfechas, sino sujetos titulares de derechos a determinadas prestaciones y conductas. Por otro lado, el nuevo paradigma otorga precisión del alcance de las obligaciones de los Estados frente a los principales derechos humanos involucrados en una estrategia de desarrollo tanto económicos, sociales y culturales, como civiles y políticos (Abramovich, 2006). 
Con dicho enfoque el Estado comienza a considerar a la juventud como un actor central para el diseño de las estrategias de desarrollo de las sociedades, generando políticas juveniles orientadas a la formación del capital humano y social y a la reducción de las brechas de desigualdad. A través del desarrollo de capacidades y conocimientos necesarios para enfrentar los desafíos de las innovaciones tecnológicas y del campo productivo, el Estado promueve que los jóvenes ingresen al sistema educativo y/o laboral, y los visualiza como un actor protagonista en el desarrollo de sus comunidades.

3. Juventudes, TIC y nuevas formas de lectura.

Pensar las juventudes y sus vinculaciones con la lectura en el presente, lleva también a considerar las TIC. De hecho Ortiz y Rubino (2010) sostienen que frente a la aparente "crisis de lectura" adjudicada a las TIC, la cual afectaría principalmente a las juventudes (una interrelación desde el paradigma joven como etapa problema, señalado en apartado anterior), se debe comprender que no ha habido una desaparición de la lectura sino una transformación posibilitada por ellas. Al respecto Morduchowicz (2009) explica que si en el siglo XV, con la invención de Guttenberg, se pasó de la cultura oral a la escrita; en el siglo XX de la cultura de la palabra a la de imagen; en siglo XXI se ha pasado de la lectura lineal a la percepción simultánea. En consecuencia ha habido un gran cambio en las modalidades y soportes de lectura, lo cual no necesariamente implica una crisis en términos negativos. Es decir, las TIC posibilitaron otros modos de leer, diversificando los formatos y las nociones clásicas de tiempo y espacio para realizar lecturas.

Pero a esta nueva cultura comunicacional (Castell, 2007) no acceden ni forman parte del mismo modo los diversos sectores socioeconómicos y generacionales de nuestra sociedad. Como bien señalan Benítez Larghi y Lemus (2012) son claras las diferencias en las oportunidades, niveles y calidad de acceso, sentidos y representaciones respecto a las TIC entre los jóvenes provenientes de los sectores populares y las clases medias y altas. En este sentido, Pittaluga (2010) sostiene que los niveles de apropiación de las TIC están ligados al sector social, la trayectoria individual y familiar.

Además, está claro que los consumos culturales juveniles, como acceso a libros e historietas, programas de tv que miran, páginas y redes sociales que utilizan, música y películas que eligen, participan en la construcción de las identidades juveniles (Reguillo, 2000).

En el presente artículo se pretende señalar ciertas particularidades en torno a las políticas locales de promoción de la lectura hacia las juventudes. Es por ello que se vuelve fundamental que los gobiernos locales, por su capacidad de intervención en el territorio, estén atentos a estas cuestiones a la hora de diseñar e implementar sus estrategias.

Una política local destinada a las juventudes debe considerar que el acceso a bienes y productos (culturales en este caso) son claves en la conformación de la cultura juvenil y la visibilidad de los jóvenes como actor social. Si los municipios desconocen y no se acercan a las particularidades de los consumos culturales de los que acceden y quedan excluidos, las juventudes difícilmente reconozcan la multidimensionalidad de sus lenguajes, lecturas y escrituras.

Una de las más importantes líneas teóricas respecto del uso de las tecnologías y las diferencias entre las experiencias de los jóvenes respecto de los adultos, permite reflexionar sobre las complejidades a la que asiste 
el Estado como actor interesado en abordar esta cuestión. Prensky (2001) y Piscitelli (2005) distinguen entre nativos e inmigrantes digitales en función de las diferentes formas de relacionarse con las tecnologías. Afirman que los jóvenes y los adolescentes de hoy son la primera generación que han crecido y se han formado con un vínculo directo y permanente con las tecnologías, generando formas de procesar la información y reflexionar, muy distintas a la del mundo adulto. Esto último genera que los "inmigrantes digitales adultos" no entienden este idioma resquebrajando el vínculo con las juventudes. La consecuencia central se vislumbra en el campo educativo, donde según datos centrales en materia de educación a nivel país, si bien se ha logrado pasar el analfabetismo de un 2,6\% a 1,9\%, siendo el segundo más bajo en la región, por problemas en materia de trayectorias educativas inconclusas y exclusión del sistema de la población juvenil, principalmente de las clases bajas y media-baja. En los establecimientos estatales, 7 de cada 10 adolescentes no terminan la secundaria a tiempo. Si bien se logró que el gasto consolidado en Educación, Ciencia y Tecnología represente un 6,5\% del PBI y que en los últimos diez años la matrícula del nivel medio creciera un 12,6\%, lo que significó que se sumaron 428.618 alumnos nuevos, esto no se tradujo en que permanecieran en los colegios. Sólo un 50\% del total de estudiantes secundarios accede a ese título y en el nivel superior sólo el 10\% de los inscriptos logra recibirse (UNESCO, 2015).

Por su parte, y retomando a Prestes (2009), este autor discute la categoría de "nativos" para llamar la atención sobre aquellas miradas que pretenden ubicar a los jóvenes como meros productos de la influencia tecnológica sin considerar los contextos sociohistóricos y vinculares:

Sabemos por experiencia que navegan solos. Son la Generación de la Red, de la Interactividad, del Downloading, del Instant Messaging, del MSN, pero sobre todo son la Generación del Naufragio. Nos han dicho que parece ser que la modernidad se hundió y con ella han perecido ideales, referencias, buques insignia y... capitanes. Estos jóvenes han aprendido a navegar solos porque han quedado solos, porque los hemos dejado solos (náufragos tecnodependientes), mientras naturalizamos cómodamente su relación con las computadoras y nos abstenemos de influir en sus vidas. Son nativos digitales que se han echado a la mar sin rumbo ni capitán, con sus rudimentarias herramientas como guías para el océano infotoxicado, en buena medida porque la generación anterior se ha abstenido de participar y eso ha generado una relación con la tecnología muy cercana. (Prestes (2009) citado en Pini et al., p. 49)

A su vez, dicho autor alerta que si bien los jóvenes se mueven "cómodamente en el mundo de la tecnología", ello no significa que sean tecnológicamente capaces o hayan desarrollado el conocimiento digital que los adultos asignan. Aquí aparecen nuevamente las profundas brechas que genera el impacto de los niveles socioeconómicos en materia de distribución y penetración digital, especialmente entre los jóvenes de diferentes estratos socioeconómicos. En nuestro país, la difusión, uso y apropiación de las TIC, está segmentada en brechas marcadas por diferencias de ingresos, educación, distribución geográfica y etnia, tal como lo demuestra los resultados de la Encuesta Nacional de Consumos Culturales y Entorno Digital llevada adelante por el Sistema de Información Cultural de la Argentina (SInCA) en el año 2013. Si bien existe una ventaja de los jóvenes respecto a otros grupos etarios en cuanto a la utilización de Internet respecto de otros grupos etarios, la segmentación por niveles de ingreso sigue siendo importante. Asimismo se encontró una correlación positiva entre el nivel educativo de la familia y el uso de las TIC.

Carlson (2010) afirma que los jóvenes saben más de ser consumidores que de ser ciudadanos, ya que hay un gran desconocimiento sobre el mundo de posibilidades en materia de información y conocimiento que habilitan 
las TIC.

Ante este panorama Cristancho et al. (2008) plantean a la democratización digital como:

Una necesidad ineludible que requiere, en primer lugar, explicitar una zona de agenda pública en el cruce entre los jóvenes y las TIC, a fin de orientar en alguna medida la apropiación de las TIC por parte de los jóvenes (...) a fin de potenciar su dimensión formativa (...). Por otro lado, contar con un criterio de democratización del acceso, para procurar mayor igualdad en formación de capacidades y oportunidades. El criterio no puede centrarse en la innovación de la frontera del conocimiento, pues a esa zona solo llegan jóvenes de alto capital educativo que rara vez vienen de hogares de menores ingresos, quedando fuera los segmentos más jóvenes entre los jóvenes. (Cristancho et al. 2008, p. 132)

Sin embargo para lograr un avance consistente en la democratización digital, en particular hacia la población juvenil, primero es necesario que los Estados locales incorporen la temá- tica en la agenda, como mencionáramos en apartados anteriores, en clave de generación, clase y género. El acceso y utilización de las TIC por parte de los jóvenes no se manifiesta de igual manera en cada uno de ellos a lo largo y ancho del país. Por tal motivo, la comprensión de esto en términos de política pública, no debe traducirse sólo en la distribución masiva de soportes digitales (fundamentalmente programas nacionales y provinciales) sin su contraparte de capacitación y formación, o en la proliferación de puntos de WIFI gratuitos en espacios e instituciones públicas (principalmente programas municipales) en zonas donde la mayoría de la población beneficiada ya posee acceso privado a la red.

En materia de promoción de la lectura es fundamental que el Estado local pueda establecer diálogos intergeneracionales que permitan definir en conjunto con los sectores juveniles la importancia de qué, cómo y para qué leer. En consecuencia, las políticas estatales que abordan la cuestión juvenil deben abandonar los sesgos universalizadores, tradicionales, conservadores y adultocéntricos que visualizan al joven como un problema a través de programas de control, represión, y de mera formación académica o laboral. Las juventudes son diversas, son sujetos de derecho a los que las condiciones socioeconómicas y de género los impacta a su vez en forma diferente. Si el Estado desconoce quiénes y cómo son las y los jóvenes que habitan la ciudad y cuáles son los principales temas que los motiva o atraviesa, difícilmente puedan avanzar en un abordaje integral de la cuestión juvenil.

\section{Reflexiones finales}

Se ha puesto de manifiesto en este texto, la existencia de brechas digitales que atraviesan gran parte de las juventudes en nuestro país. Asimismo, se han mostrado los nuevos roles que atraviesan los municipios en la dinámica del abordajes de la cuestión social y juvenil.

Continúa pues el desafío de que los Estados locales asuman el abordaje de la difícil ecuación entre TICJuventudes-Desigualdades-Promoción a la lectura.

En este sentido, la adopción del enfoque de derechos expuesto más arriba, permite dotar de una mirada más amplia, integral y desde el territorio nuestra problemática. Este momento puede ser caracterizado como de Descentramiento, en el que el saber traspasa los límites de exclusividad otorgados por los libros y la escuela ya 
que circula por otras esferas como la comunidad virtual. A su vez, se vive una situación de Destemporalización en las que el tiempo y momento legitimado de la distribución y aprendizaje del saber se han expandido, ya no es sólo a una corta edad y en la escuela, sino que en todo momento, sin necesidad de un lugar propio y a lo largo de toda la vida (Barbero, 2003). En este sentido, el acceso a la lectura de libros es un punto de partida para otras alfabetizaciones.

El desafío está en formar jóvenes ciudadanos que también sepan leer noticieros de tv, periódicos o hipertextos informáticos. Es decir, acceder a la multiplicidad de escrituras y discursos desde donde se definen decisiones claves que lo afectan política, económica, familiar y laboralmente (Morduchowicz, 2009). Esto implica también poder aprender a elegir en medio de semejante universo informativo; determinar la confiabilidad de la fuente; poder dudar, discernir, cotejar y evaluar distintas versiones; saber navegar estratégicamente en la red y; disponer de estrategias de lectura y escritura virtual (Ortiz y Rubino, 2010, p. 2). Las comunidades virtuales pueden generar procesos de aprendizaje tanto como la comunidad académica.

Pero para ello es necesario ampliar el conjunto de acciones "tradicionales" de los municipios en materia de promoción de la lectura fuertemente vinculada, como ya se ha resaltado, con la instalación de bibliotecas públicas y centros de apoyo escolar. En este sentido, se abre un campo de acción y estudio poco desarrollado por los gobiernos locales de Argentina, que consiste en la implementación de políticas locales que complementen las políticas educativas y el accionar de las escuelas (principalmente en manos de las provincias) en materia de promoción de la lectura, teniendo en claro los nuevos formatos de lectura y fundamentalmente la potencia liberadora y creadora de ésta.

Con este marco conceptual, la política pública juvenil se orienta a la generación de condiciones para que los jóvenes puedan autorealizarse y a su vez puedan participar en la construcción de la realidad social en la que se insertan. Esta noción se relaciona directamente con el enfoque de derechos. La adopción de este paradigma determina tres principios centrales que atravesarían el diseño e implementación de las políticas públicas juveniles de todo Estado: el principio de inclusión y los estándares sobre igualdad y no discriminación; el principio de participación y su relación con los derechos civiles y políticos; y el principio de rendición de cuentas y responsabilidad y los mecanismos para hacer exigibles los derechos.

Notas

1. Este trabajo se inscribe en un trabajo de investigación más amplio en el marco de una beca doctoral de CONICET denominada "Desarrollo de sistemas de protección social y bienestar juvenil en el ámbito municipal de la provincia de Córdoba".

2. Cravacuore (2007) señala principalmente deficiencias en materia administrativa, organizativa, presupuestaria, de personal profesionalizado y en el diseño, gestión y evaluación de políticas públicas.

3. El concepto de juvenilización es definido por el autor como el proceso de construcción de identidades e imaginarios sociales a partir de los modelos de ser joven que existen en nuestras sociedades.

Bibliografía 
Abramovich, V (2006). "Una aproximación al enfoque de derecho en las estrategias y políticas de desarrollo". Revista de la CEPAL, № 88, pp. 35-49.

Arroyo, D (1997). "Estilos de gestión y políticas sociales municipales en Argentina”. En García Delgado (comp.) Hacia un nuevo Modelo de Gestión Local, Municipio y sociedad civil en Argentina. Buenos Aires: FLACSO Oficina de Publicaciones del CBC, UBA - Universidad Católica de Córdoba.

Balaguer Prestes, R. (2009), Ni nativos ni inmigrantes: Ilamadles náufragos digitales. (En línea). Blog de Cultura Digital de la UBA. http://ubaculturadigital.wordpress.com/2009/02/27/ ni-nativos-ni-inmigrantes-llamadlesnaufragos-digitales.

Balardini, S. (2000). "De los jóvenes, la juventud y las políticas de juventud”. En Última Década №13. Viña del Mar: CIPDA. Disponible http://www.scielo.cl

Barbero Martín, J. (2003). La educación desde la comunicación. Buenos Aires: Grupo Editorial Norma.

Benítez Larghi, S., \& Lemus, M. (2012). Juventudes, desigualdades y TIC. In VII Jornadas de Sociología de la UNLP 5 al 7 de diciembre de 2012 La Plata, Argentina. Argentina en el escenario latinoamericano actual: Debates desde las ciencias sociales. Universidad Nacional de La Plata. Facultad de Humanidades y Ciencias de la Educación. Departamento de Sociología.

Bombini, G. (2008). La lectura como política educativa. Revista Iberoamericana de Educación, № 46 p. 19-35, Disponible en: http://www.rieoei.org/

Bourdieu, P. (2002). La "juventud" no es más que una palabra. En Sociología y Cultura, pp. 163-173. México: Grijalbo, Conaculta.

Braslavsky, C. (1986). La juventud argentina: informe de situación. Buenos Aires: Biblioteca política argentina. Centro de editor de América Latina.

Calabre, L. (2009). Políticas culturais: reflexões e ações. São Paulo: Itaú Cultural.

Calado, P. M. (2012). Las políticas culturales de los gobiernos locales en la argentina. Pueblos y fronteras, 7(13), $127-146$.

Carlsson, U. (ed.) (2010). Children and youth in the digital media culture. From a Nordic horizon. University of Gothemburg. Suecia: NORDICOM.

Castells, M. (2007). Communication, power and counter-power in the network society. International journal of communication, 1(1), 29.

Chaves, M. (2005). “Juventud negada y negativizada: representaciones y formaciones discursivas vigentes en la Argentina contemporánea" en Última Década № 13, Valparaíso: CIDPA. Disponible http://www.scielo.cl

CIPPEC (2005). Hacia la Planificación Estratégica. Buenos Aires: Fundación Cippec - Fundación Innova. 
Cravacuore, D. (2007). “Los municipios argentinos (1990 - 2005)”. En: Cravacuore, D. e Israel, R. (comp.) Procesos políticos municipales comparados en Argentina y Chile (1990-2005), pp. 25-49. Editorial de la Universidad Nacional de Quilmes - Universidad Autónoma de Chile. Buenos Aires.

Cristancho, C.; Guerra, M. y Ortega, D. (2008). “La dimensión joven de la conectividad en América Latina: Brecha, contextos y políticas". En Revista Pensamiento Iberoamericano, №3, pp. 117-138.

García Delgado, D. (1997). “Introducción: Nuevos escenarios locales. El cambio del modelo de gestión”. En García Delgado (comp.) Hacia un nuevo Modelo de Gestión Local, Municipio y sociedad civil en Argentina, pp. 13-42. Buenos Aires: FLACSO - Oficina de Publicaciones del CBC, UBA - Universidad Católica de Córdoba.

Golbert, L. (2004). ¿Hay opciones en el campo de las políticas sociales? El caso del gobierno de la Ciudad Autónoma de Buenos Aires. Buenos Aires: CLACSO.

Krauskopf, D. (2000). “Dimensiones críticas en la participación política de los jóvenes”. En Balardini, S. La participación social y política de los jóvenes en el horizonte del nuevo siglo. Ciudad Autónoma de Buenos Aires: CLACSO. Disponible en http://bibliotecavirtual. clacso.org.ar

Maceira y otros (2010). El papel de los municipios en la inversión pública social en infancia y adolescencia: Resultados de una encuesta nacional a municipios argentinos. Buenos Aires: Fundación Cippec - Unicef.

Margulis, M. (2001). “Juventud: una aproximación conceptual”. En Donas Burak (comp). Adolescencia y Juventud en América Latina, pp. 41-56. Costa Rica: Libro Universitario Regional (EULAC-GTZ).

Mihal, I. (2009). Plan Nacional de Lectura: notas sobre una política de promoción de la lectura. Revista Pilquen (11). Disponible en http://www.revistapilquen.com.ar/Ciencias Sociales/Sociales11/11_Mihal_Plan.pdf

Mihal, I. (2012). Saberes, concepciones y prácticas acerca de los actores que gestionan las políticas culturales de lectura. Horizontes Antropológicos, 18 (38), pp. 287-311.

Morduchowicz, R. (2009). "Cuando la educación en medios es política de Estado". Comunicar N³2, vol XVI, Revista Científica de Educomunicación, pp. 131-138.

Ortiz, M. V., \& Rubino, L. S. "Las TICs y las nuevas formas de lectura". Revista Boradores vol X/XI. Universidad Nacional de Río Cuarto, Córdoba, 1-15; disponible en http://www.

unrc.edu.ar/publicar/borradores/Borradores.htm.

Pini, M.; Musanti, S.; Kaufman, G. \& Amaré, M. (2012). Consumos culturales digitales: jóvenes argentinos de 13 a 18 años. Buenos Aires: Educ. ar SE, Ministerio de Educación de la Nación. Disponible en http://bibliotecadigital.educ.ar/uploads/contents/TIC_Con sumosCulturalesPARAokFINAL1.pdf

Piscitelli, A. (2005). "Epistemología de las marcas en la era de la incertidumbre. La generación arroba". En Portal educ.ar, Debates, Educación y TIC, Nuevos alfabetismos. Disponible en http://portal.educ.ar/debates/educacionytic/nuevos-alfabetismos/ epistemologia-de-lasmarcas-en-la-era-de-laincertidumbre-la-generacion-arroba.php 
Pittaluga, L.; A. Rivoir; S. Baldizán; S. Escuder y F. Di Landri. (2010). Informe de Investigación "El Plan Ceibal: Impacto comunitario e inclusión social 2009 - 2010". Montevideo. ObservaTIC, Facultad de Ciencias Sociales, Comisión Sectorial de Investigación Científica, Universidad de la República.

Prensky, M. (2001). "Digital natives, Digital Immigrants”. En On the Horizon, Vol. 9, N. ${ }^{\circ}$ 5. NCB: University Press. http://www.marcprensky.com/writing/ Prensky\%20-\%20Digital \%20Natives,\%20Digital\%20Immigrants\%20\%20Part1.pdf.

Quapper, K. (2001). “¿Juventud o juventudes? Acerca de cómo mirar y remirar a las juventudes de nuestro continente". En Donas Burak (comp.) Adolescencia y Juventud en América Latina, pp. 57-74. Costa Rica: Libro Universitario Regional (EULAC-GTZ).

Rebón, M. (2011). "El estudio de la institucionalidad de las políticas culturales". Anuario de Indicadores Culturales, pp. 232-239. Disponible en http://untref.edu.ar/documentos/ indicadores2010/El\%20estudio\%20de\%20la\%20institucionalidad\%20de\%20las\%20po liticas\%20culturales\%20de\%20los\%20gobiernos\%20locales\%20Marcela\%20Rebon.pdf

Reguillo, R. (2000). Emergencias de las culturas juveniles. Estrategias del desencanto. Buenos Aires: Norma.

Rodríguez, E. (2000). “Juventud y políticas públicas en América Latina: experiencias y desafíos desde la gestión institucional”. Última Década N¹3, pp. 25-58. Viña del Mar: CIDPA. Disponible http://www.scielo.cl

Rosanvallon, P. (1995). La nueva cuestión social. Buenos Aires: Manantial.

Saltalamacchia, H. (1990). "La juventud hoy: un análisis conceptual”. Revista Ciencias Sociales. Disponible en http://saltalamacchia.com.ar

Abstract: This article discusses a series of statements about the supposed "reading crisis" in the youths; The classical views on what, how and when young people should read and about the most classic and conservative paradigms on how to define and address the issue of youth from the State. From this, the central objective is to give relevance to the capacity of the local States in the approach to ICTs to intervene in the promotion of reading, implementing youth public policies that are oriented to the generation of conditions so that the young can be autorealized already In turn they can participate in the construction of the social reality in which they are inserted.

Key words: Youth - ICT - Promotion of Reading - Local State - Public Policies.

Resumo: Este artigo discute uma série de declarações sobre a alegada "crise de leitura" na juventude; as opiniões clássicas sobre o que, como e quando os jovens devem ler e sobre os paradigmas mais clássicos e conservadores sobre como a questão da juventude é definida e abordada pelo Estado. Com base nisso, o principal objetivo é dar relevância à capacidade dos Estados locais na abordagem das tecnologias da informação e da comunicação (TIC) para intervir na promoção da leitura implementando políticas públicas de juventude orientadas para a geração de condições para que os jovens se auto-realizam e, ao mesmo tempo, participar da construção da realidade social em que estão inseridos. 
Palavras chave: Juventude, Tecnologias da informação e da comunicação, Promoção de leitura, Estados locais, Políticas públicas.

[Las traducciones de los abstracts al inglés y portugués fueron supervisadas por el autor de cada artículo]

Juventudes y TIC: Estados locales frente al abordaje de la promoción de la lectura fue publicado de la página 135 a página150 en Cuadernos del Centro de Estudios de Diseño y Comunicación N²72 